\title{
Influence of long-term intravenous drug use on physiological indicators and morphological changes of internal organs in piglets
}

\author{
M.I. Barashkin, I.M. Milshtein, M.M. Sibiryakov, A.S. Barkova*, and E.S. Eroshenko \\ The Ural State Agrarian University, Yekaterinburg, Russia
}

\begin{abstract}
The article considers the state of physiological indicators and morphological changes of internal organs during the application of longterm non-inhalation narcosis in pigs of two groups while providing artificial lung ventilation.
\end{abstract}

\section{Introduction}

Any operation intervention will require the surgeon to provide a high-quality, safe anesthesiology, which has the least adverse effect on the animal, but at the same time it is most effective under conditions in this or that surgical manipulations. There are a lot of different kinds of anesthetics and annotations of anesthesia for various conditions, time and minimum risk to animal health, such as inhalation anesthetics and non-inhalation (injection ones) [1].

It should be understandable, that pigs are "technological" animals, which means they almost don't have any surgical manipulations at all, except routine procedures, due to low economic benefits. Pigs, however, is the one of the best biological models of human beings, therefore has a big importance and interest into an experimental branches of medicine, that explains a necessity of explore a new adequate scheme of general anesthesia [7,8].

The main aim of the work was an influence evaluation of intravenous anesthesia with physiological indicators and morphological changes of internal piglets' organs while modeling of atalectasis and providing of artificial lung ventilation.

To achieve this objective, the following tasks were set:

1. Compare the two schemes of intravenous anesthesia in piglets

2. Investigate influence and direct changes of physiological piglets rates during providing of a long-term non-inhalation anesthesia

3. To find out how non-inhalation anesthesia influent on respiratory and cardiovascular systems of piglet in a state of atelectasis, with artificial lung ventilation providing.

4. Find out practical recommendations for non-inhalation anesthesia while doing an operational intervention of biological models.

The relevance of the study has two main factors. Firstly, anatomy-physiological specifies of pigs, which are very similar with human ones and make them almost perfect laboratory

\footnotetext{
* Corresponding author: huretan@gmail.com
} 
animals, moreover, huge quantity of medical experiments usually involve a prolonged state of narcosis, especially with lung manipulations, because this case should be conducted with intravenous narcosis only. Therefore it's very necessary to create a certain scheme, which will provide being animal into narcosis statement for pretty long time, but at the same time will be safe as much as it can and provide rapid recovery from the condition of narcosis. Second, it should be taken into account, that a legislative frame low of Russian Federation, which in turn restricts the use of group A drugs (a list of drugs and poisons) for experimentations and investigations, and this certain fact was a reason for producing of experimental schemes for prolonged kind of pigs' narcosis.

\section{Materials and methods}

All investigations were considered on a basis of Department of Obstetrics and Gynecology of Ural State Agrarian University, Animal rehabilitation centre of Ural State AU together with Maternal and Infant Health Research Institute (MIHRI) of RF Ministry of Health and SHVABE LLC Yekaterinburg from 2016 till 2020 years.[2]

Since October 2016 the Animal Rehabilitation Centre of Ural State Agrarian University, has been holding international, scientific-practical conferences and master-classes in the frame work of which a techniques and methodology of non-inhalation drug addiction of pigs were practiced with the participation of OOM Ministry of health of Russian Federation [3].

The subjects of a study were piglets of 18 month from 3 to 4 kilos. For this period of experiments (during 4 years), 29 heads of piglets were administered by non-inhalation narcosis and 10 sub-headings aged 5-6 month weighing between 12-19 kilos for complicated intravaginal hernia. All animals were clinically observed, the result was positive enough - clinically healthy, except for a group of animals with intravaginal hernia.

Ten piglets of large white breed was studied various schemes of anesthesia and discovered just one appropriate, which was successfully applied during Maternal and Infant Health Research Institute of RF Ministry of Health in 2015.

The animal toilet was used before conducting the manipulation. After onset myorelaxation (where did it come from?) an intravenous catheter $26 \mathrm{G}$ was installed into external vein of the auricle, which was fixed by a Band-Aid. Veins were squeezed at the base, after blood filling, catheter easily moved forward. Pigs with light skin coloring have very clear auricle veins visualization. For predicting of a spontaneously respiratory arrest and providing of artificial lungs ventilation the animal was intubated. Piglet was fixed into a thoracic position, for the well visualization of a throat was used laryngoscope with the curved blade. The endotracheal tube was bent down to a voice slot. At this point the tube was rotated 180 degrees along it's longitudinal axis and gently pushed her through the trachea.

When the tube was pushed through the trachea on 1-2 sm it was rotated again by 180 degrees to inject it into the trachea further. The tube was fixed by ligature to the upper jaw and cuffs inside the trachea [6].

The histological study was carried out according to a generally accepted method, followed by the coloration of the lower section with hematoxylene and eosin on Van Gieson.

\section{Research results}

For achievement of prolonged non-inhalation narcosis was two complex circuits have been tested to achieve long-term non-inhalation narcosis. 
The first scheme of anesthesia is shown in the table number 1 .

Table 1. The first scheme of non-inhalation narcosis.

\begin{tabular}{|c|c|c|}
\hline $\begin{array}{c}\text { Method of } \\
\text { injection }\end{array}$ & Medicine & Dosage, $\mathrm{ml}$ \\
\hline Intramuscular & Atropin & 0,1 \\
\hline Intramuscular & Ksilazin & 0,3 \\
\hline Intramuscular & Zoletil 100 & 0,3 \\
\hline Intravenous & Propofol $10 \mathrm{Mr} / \mathrm{MJ}$ & 0,5 \\
\hline Intravenous & $\begin{array}{c}\text { Zoletil } 100 \mathrm{in} \text { breeding } 1: 9 \text { with } \\
0,9 \% ~ N a C l\end{array}$ & $50 \mathrm{ml}$ at a speed of $15 \mathrm{ml} / \mathrm{h}$ \\
\hline Intravenous & $\begin{array}{c}\text { Propofol } 10 \mathrm{mg} / \mathrm{ml} \text { in breeding } \\
1: 4 \text { with } 0,9 \% ~ N a C l\end{array}$ & $50 \mathrm{ml}$ at a speed of $15 \mathrm{ml} / \mathrm{h}$ \\
\hline
\end{tabular}

Graph of physiological performances on the scheme №1

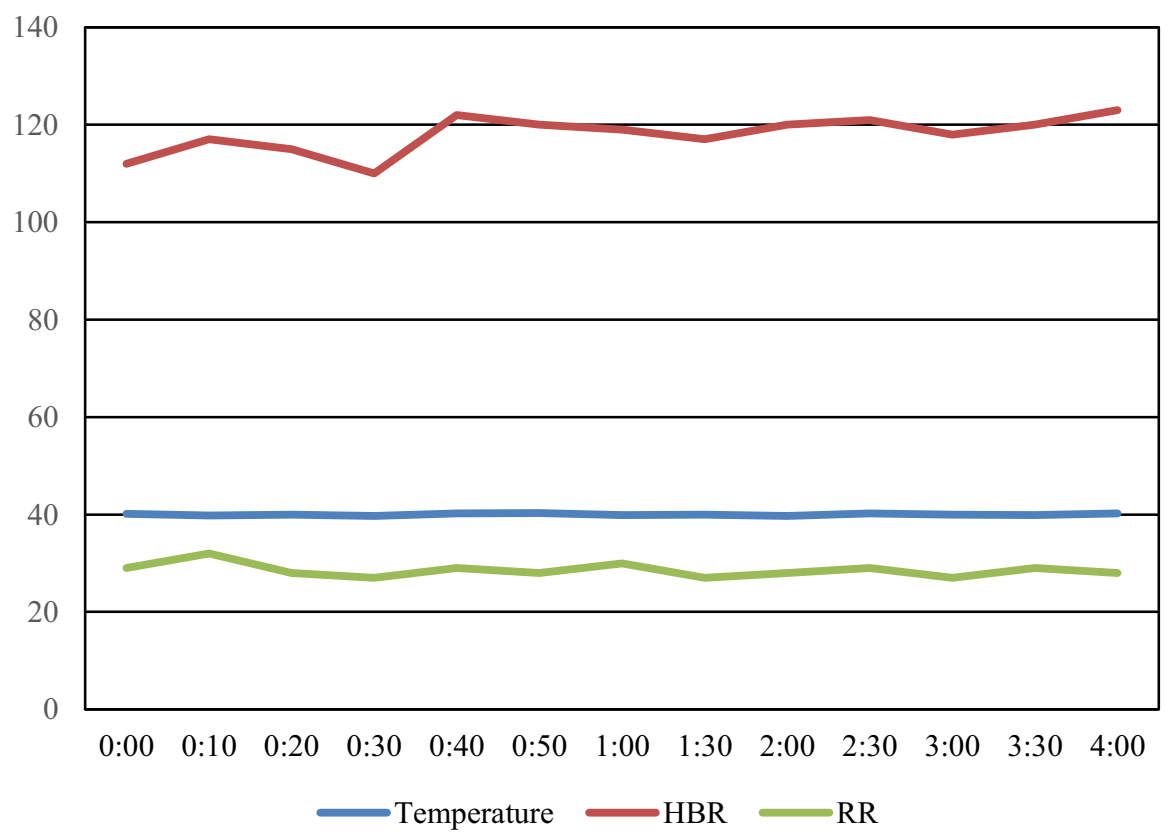

Fig. 1. Physiological readings of piglets using the first anesthesia scheme.

As a result of applying this scheme were noticed minor deviations from the norm among clinical performances. The opportunity to provide the intubation appears between 10-12 minutes. The time for narcosis reached for 8 hours, the post-anaesthetic period was 1 hour.

The second scheme of anesthesia is shown in table 2.

Table 2. Second scheme of non-inhalation narcosis.

\begin{tabular}{|c|c|c|}
\hline $\begin{array}{c}\text { Method of } \\
\text { injection }\end{array}$ & Medicine & Dosage,ml \\
\hline Intramuscular & Atropine & 0,1 \\
\hline Intramuscular & Meditin & 0,2 \\
& Dimedrol & 0,2 \\
\hline
\end{tabular}




\begin{tabular}{|c|c|c|}
\hline Intramuscular & Zoletil 100 & 0,05 \\
\hline Intravenous & $\begin{array}{c}\text { Propofol } \\
10 \mathrm{Mmg} / \mathrm{ml}\end{array}$ & 0,5 \\
\hline Intravenous & $\begin{array}{c}\text { Zoletil } 100 \mathrm{in} \\
\text { breed } 1: 9 \text { with } \\
0,9 \% \mathrm{NaCl}\end{array}$ & $\begin{array}{c}50 \mathrm{ml} \text { at a speed } \\
\text { of co } 15 \mathrm{ml} / \mathrm{h}\end{array}$ \\
\hline Intravenous & $\begin{array}{c}\text { Propofol } \\
10 \mathrm{mg} / \mathrm{ml} \text { in } \\
\end{array}$ & $\begin{array}{c}50 \mathrm{ml} \text { at a speed } \\
\text { of } 15 \mathrm{ml} / \mathrm{h}\end{array}$ \\
& $\mathrm{NaCl}$ & \\
\hline
\end{tabular}

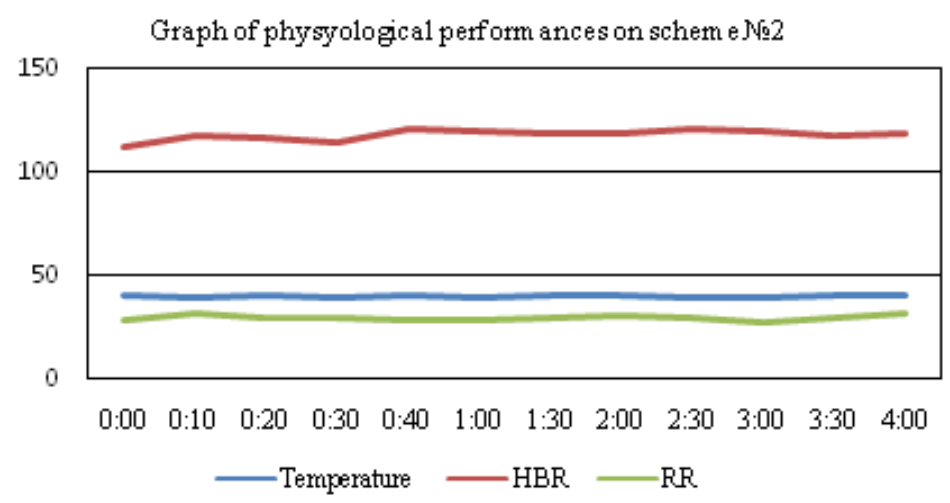

Fig. 2. Physiological indicators of using the second scheme in pigs.

While using the second scheme of narcosis were noticed a little deviations from the norm among clinical indicators as well, at the same time the intubation possible in 8-9 minutes. The total narcosis duration was 8 hours, post anesthetic sleep - 1 hour.

Comparing two schemes of intravenous anesthesia was conducted analysis of impact "Ksilazine" and "Meditin".

These are a drugs of an antagonists group $\alpha 2$-adrenoreceptors, their pharmacological properties are similar, but "Meditin" is more modern and narrowly directed drug for sedation, because it doesn't influent on $\alpha 1$ adrenoreceptors, making more side effects, more over medetomerine being as a main active substance of the drug "Meditin", potentiates the action of zoletyl. «Meditine» $40 \mu \mathrm{g}$ of Medetomin per 1 kilo body weight $(0,04 \mathrm{ml})$ with $5 \mathrm{mg}$ Zoletyl per $\mathrm{kg}$ of body weight causis anesthesia the same prolongation, that $1 \mathrm{mg}$ of Ksilazine per one kilo $(0,05 \mathrm{ml})$ with $20 \mathrm{mg}$ of zoletil per $\mathrm{kg}$. One more advantage of "Meditin" is that it has the antagonist "Antimedine", which applied with the purpouse of eliminating sedative action of medetomin in cats and dogs, also when it'll be necessary to eliminate a side effect of medetomine. Atypamesol - the main active substance a presynaptic $\alpha-2$ antagonist, highly specific to the presynaptyc $\alpha$-2-adreno receptors, blocks them, resumes impulse transmission.

In the course of the study were investigate and compared two schemes of non-inhalation anesthesia in pigs and influence intravenous anesthesia on morphological piglet's lung statement which have aspiration liquid, while providing of artificial lung ventilation.

After the providing of non-inhalation anesthesia were selected tissues of the organs (lungs and heart) for conducting of histological research. The main task of a study was evaluating of morphological lung statement during the atelectasis.

The effect of asphyxiation on heart and vessels is very pronounced from the very beginning of the asphyxiation. In to a normal conditions blood flow through the lungs is 
caused not only by the activity of right cardiac ventricle, but also by respiratory movements. Every breath expands the airways and cavities, as well as the blood vessels in them, thereby implanting blood from the heart. When exhaled, the capillaries contract, contributing to the pushing of blood into the left atrium and ventricle. When breathing slows or stops blood circulation in the lungs breaks: the right ventricle cannot push the entire blood mass through the lungs. Respiratory shortness of breath creates conditions lungs overwhelming with blood, and they can't easily release it. Therefore the right cardiac half overflow with blood, which it can't push through the overcrowded lungs. The venous vessels leading to the heart, also rapidly overflowing by blood. This condition is clearly visible in the jugular vein. Vessels of a head also rapidly overflowing with blood. The blood becomes dark red colored with a blue shade, asphyxiated, that could be visible in animals as a result of the mucous membranes, developing one of the most vivid sings of asphyxiation - blues (cyanosis).

In the beginning, when heart beating is getting slower, blood pressure goes down, but into a period of expiratory breath shortness it rises up again, all capillaries and veins overflow; the right cardiac part even more overflowing by blood, which it can no longer push through the lungs. Heart starts contracting more often, blood pressure drops. Exhausted from the previous work heart, supplied by asphyxial blood, continue to work even weaker, and this coincides with the phase of respiratory pause. At the period of irregular (erratic) terminal respirations, when the blood partially passes through the lungs, there is an increase and a new slowdown in the cardiac function. After a complete respiratory arrest, the activity of the heart gradually weakens and finally stops. There are some observations indicating that in some cases heart activity continued for 30 minutes after the respiration stopped.

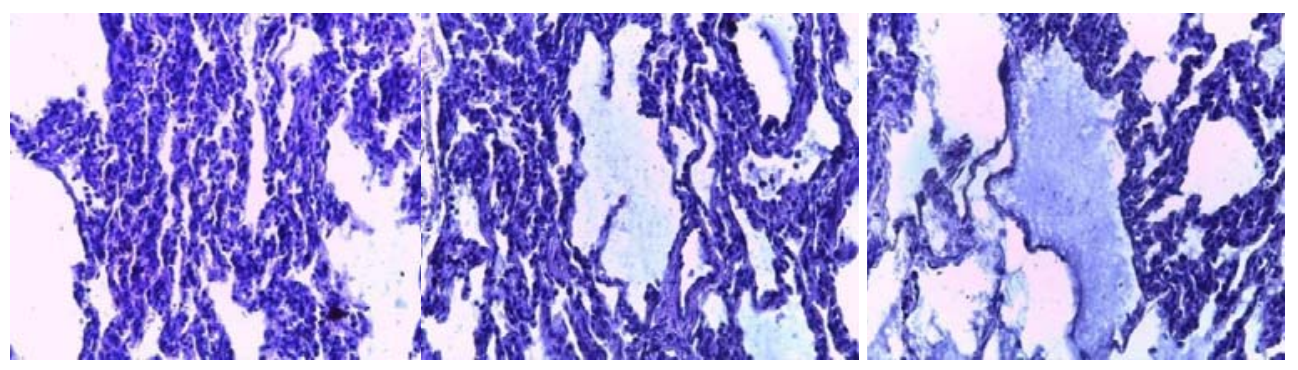

Fig. 3. Histologycal lung cuts of the head from group №1 (left to right) The heart of atelectase. Compensatory emphysema. Hot spot of edema. All images - not everywhere have right couloring and right zooming, this is how Van Gieson looks like. It is better to take pictures separately.

Lymphoid follicles into a bronchial wall indicate high local immunity and high body resistance. The compensatory emphysema develops while the lungs volume decreasing, and when the respiratory surfaces are excluded due to atelectase.

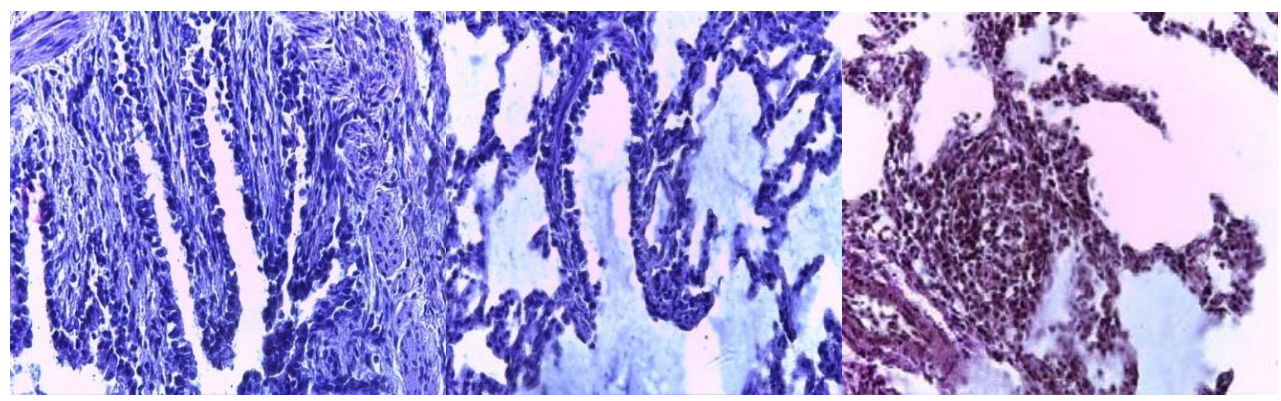


Fig. 4. Histological lung cuts of the head from group №1(left to right). Dilation of bronchial medium caliber. Small-caliber of bronchial fracture as a result of bronchoectasy. Lymphoid follicles into a bronchial wall (Van Gieson coloration).

Edema appeared because of alveocappillary membrane was directly injured. Due to the action of aspiration liquid, breaks the protein structure of the alveolacapillary membrane and fluid goes out into interstitial space.

Cased of an extensive lung atelectasis, large bronchial cavities fall, and minor ones are torn, and as a result the bronchoectasia develops.

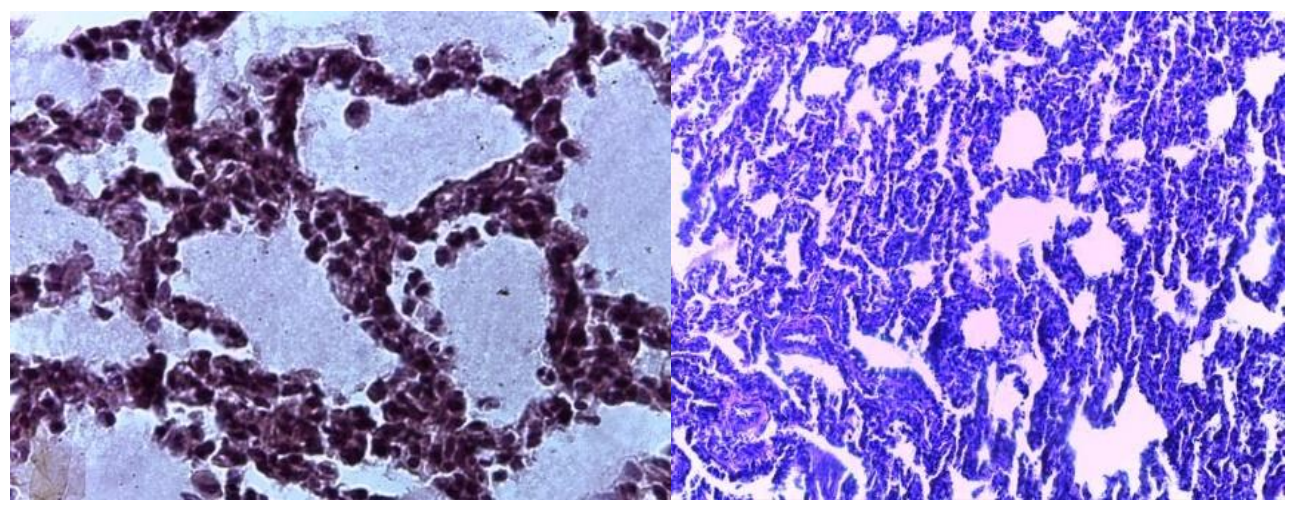

Fig. 5. Histological lung cuts of the head from the group№1 (left to right) Edema - large protein molecules (fibrinogen), exudation of cells of cardiac defects (coloration by Van Gieson). Into the caudal lobe there are significant hot spots of atelectasis.

Bronchoectasis is a pathology, which in the bronchus cavities irreversible expansions and deformations are created, which in turn provoke chronic purulent inflammation of the bronchial mucus membrane - purulent endobronchitis.

Also effusion of syderophages (cardiac disease cells) in the lung alveolis processed, pathology explained with toxic attack from the drugs on the body.[5]

These investigations says about irreversible changes into the lung parenchyma during atelectasis 3,5 hours prolonged, while being into narcosis for 5 hours long.

Changes, happened into the respiratory and cardiac systems are irreversible, and it is caused the quality of a pigs life from the group №1 noticeably decreases and requires of special living conditions: sterilized enclosed space with an ability to change a temperature and humidity whatever they should be, straw and hay has to be strictly systematically examined for fungi (each batch) and conditions of a storage must be observed as well. [5]

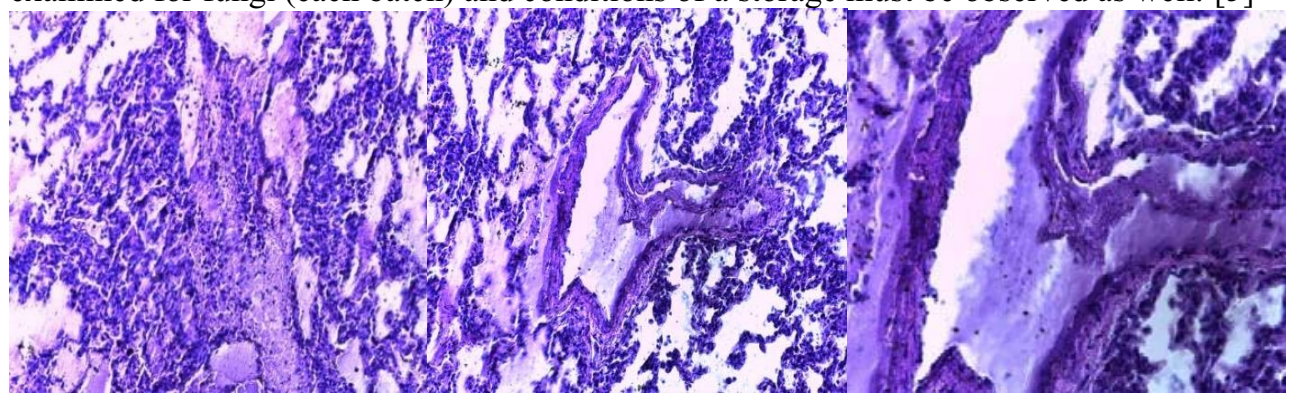

Fig. 6. Histologycal cuts of the lungs of the head from the group№2(left to right) Sygnificant amount of edema 9total edema) The blood into a vessels doesn't content erythrocytes. Swelling and melting of endotolial wall of vessels. 


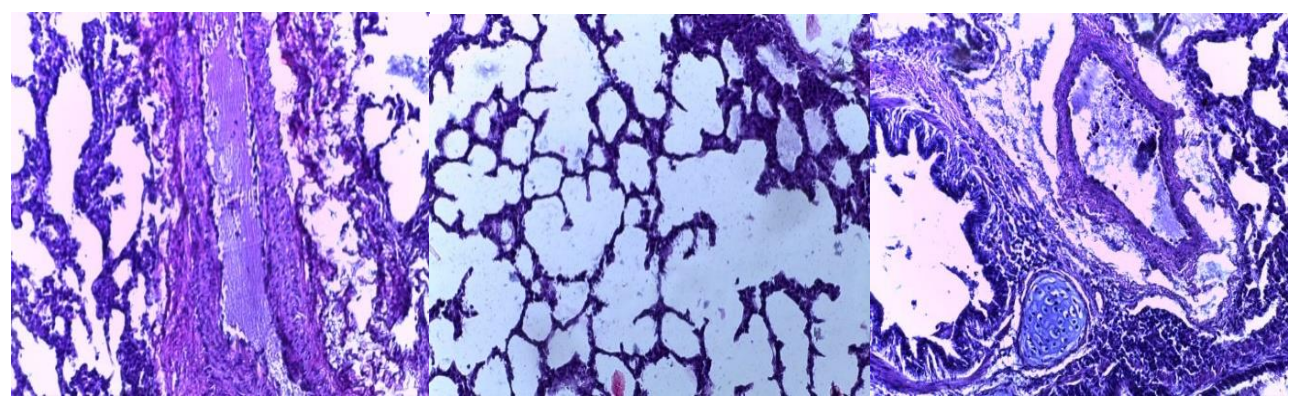

Fig. 7. Histological lung cuts of the head from the group№2 (left to right). Clot formation, changing into the vessels wall. Significant hot points of emphysema. Perevascular edema, lymphoid fallicle.

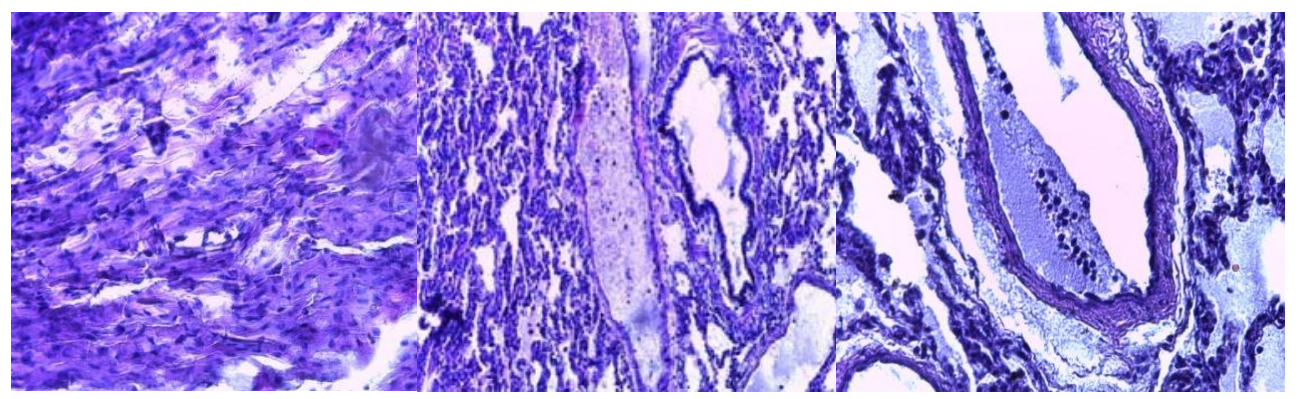

Fig. 8. Histological heart cuts of the head from the group №2 (left to right). Death of cardiomyocytes. Hemolisis of erythrocytes.

The main differences in morphological changes, happened into a body of a head from the group №2 are changes into a cardiovascular system.

As a result of oxygen failure in the vascular tract, the blood is free from erythrocytes and oxygen starvation occurs. Also due the long-term immobility, drug influences for narcosis and atelectasis, clots create in lung vessels, perivascular and peribronchial edema. Erythrocyte hemolysis occurs, this process is caused by the appearance of China-Stokes respiration, the apnea period alternates with increasing of deepness respiratory movements, which after the achievement of a certain phase, fade again. The (apnea) pause about 30-45 s. occurs, and then a new cycle begins.[4]

There is no way out from the narcosis for the pig from the group №2. Death occurs during two hours, when the sharp coronary failure begins. Duration of atelectasis is 4,5 hours, time of being into narcosis condition is 6,5 hours.

\section{Conclusion}

On the basis of obtained results we can make such a conclusions:

1. Into the process of study were developed, learnt and compared 5 schemes of noninhalation pigs anesthesia, of the presented conditions, three are suitable, each of them have a similar indicates and costs, but the most cost-effective is scheme 5 .

2. Intravenous anesthesia with a using the combination of "Propofol" and "Zoletyl100" provides a reliable level of anesthesia, but the intubation and respiratory supporting of animals are the most important conditions due to physiological and anatomical particular qualities of pigs.

4. When carrying out non-inhalation narcosis in pigs with the use of combination of "Propofol" and "Zoletyl" it is necessary to provide an adequate sedation, what will be allow doing an endotracheal intubation of trachea. But the anatomical structure of the larynx and 
the nose glow must be considered. Pigs are almost completely devoid a superfacial veins, so for intravenous access we chose an external ear vein.

5. The studied scheme could be applied not only while providing an experimental investigates, but into a veterinarian practice for complex surgery operations as well.

6. The above provides the basis for introduction an intravenous anesthesia schemes for future work together with Maternal and Infant Health Research Institute of RF Ministry Health, and practical veterinarian surgery. The work can also be considered from the point of methodological guide for intravenous anesthesia in pigs.

7. The influence of intravenous anesthesia and prolonged atelectasis onto respiratory organs and cardiovascular system depends on narcosis and atelectasis duration. Within five hours of anesthesia and four and a half hours of atelectasis, an animal can get out from a narcosis, but pathological processes rapidly reduce animal's quality of life. On a background of bronchoectasia, purulent endobronchitis develops; cells of syderophagus testify to acquire heart defect. After six and a half hours of narcosis and four and a half hours of atelectasis pathological changes provoked a death, which occurred in a result from paralysis of vascular centre. In histological research of the lungs were explore: total edema, perivascular and peribronchial edema, the vessels blood doesn't contain erythrocytes, melting of endotolial vessels wall, division of blood into white and red substances, formation of clots.

\section{References}

1. I.M. Milshtein, M.M. Sibiryakov, Biotecnological herald, 3(20), 13 (2019)

2. I.M. Milshtein, M.M. Sibiryakov, Agrarian education and science, 3, 25 (2019)

3. I.M. Milshtein, M.M. Sibiryakov, Biotecnological herald, 4(21), 14 (2019)

4. V.M. Scorlyacov, S.V. Savina, Pathologycal physiology: Brief Lectures for Third Course Students 36.05.01 Veterinary, Federal State-Funded Educational Institution of higher Education of Saratov GAU, 127 (Saratov, IC «Science», 2016)

5. V.P. Smirnov, I.A. Panysheva, Pathomorphology of cardiomyocytes in sudden cardiac death, Medicine: todays challenges: materials III International science conferention, 43-46 (Moscow, Buki-Vedi, 2016)

6. J.R. Linkenhoker, T.H. Burkholder, C.G. Linton, et al., Journal of the American Association for Laboratory Animal Science, 49(3), 344-351 (2010)

7. M.M. Swindle, A. Makin, A.J. Herron et al., Veterinary Pathology, 49(2), 344-356 (2012)

8. K. Yamada, A. Griesemer, M. Okumi, Transplantation Reviews, 19(3), 164-177 (2005)

9. J. Zeyland, D. Lipiński, R. Słomski, Journal of Applied Genetics, 56(2), 211-218 (2015) 